\title{
Intercalation In Layered Materials
}

\author{
M.S. Dresselhaus
}

\section{Introduction}

There is great current interest in layered anisotropic materials. Much of the interest relates to the possibility of an approximate decoupling of 3-dimensional (3D) behavior into $1 \mathrm{D}$ properties perpendicular to the layer planes and $2 \mathrm{D}$ properties associated with the layer planes. Since this decoupling in layered materials is enhanced by intercalation, much attention has been given to intercalation compounds in recent years as a means to study interesting questions of low dimensional physics. ${ }^{1}$ Since many deliberately structured materials also are prepared in layered sequences, there are many close connections between the properties of intercalated layered materials and deliberately structured materials. This article will focus on several important connections between intercalated layered materials and multilayered superlattice structures prepared by molecular beam epitaxy (MBE) and metalorganic chemical vapor deposition (MOCVD).

Interest in intercalation compounds has led to three lively MRS symposia on this topic (Fall 1982, Fall 1984, Fall 1986) ${ }^{2-4}$ and a number of summer schools and workshops, one of which was held in July 1986 at Erice, Sicily, under the auspices of NATO and the European Physical Society. The published lecture notes ${ }^{1}$ from this summer school provide a useful introduction and reference for students and others interested in this field.

Intercalation compounds are formed by the insertion of atomic or molecular layers of a guest chemical species between layers in a host material. This is illustrated in Figure 1 for potassium-intercalated graphite. The intercalation process occurs in highly anisotropic layered structures where the intraplanar binding forces are very strong and the inter planar binding forces are very weak. When these conditions prevail, entire layers of guest species can be inserted between layers of the host material. Examples of host materials for intercalation compounds are numerous, including graphite, transition metal dichalcogenides, III-VI layered semiconductors, some silicates, oxides and metal chlorides, clays, polymers, and gels. An even larger number of intercalant guest species have been identified. For example, several hundred different chemical species are known to intercalate into graphite. By proper choice of the host material, the intercalant species and its concentration, a large number of different intercalation compounds with interesting properties can be prepared..$^{1-6}$

For the transition metal dichalcogenides $\mathrm{TX}_{2}$ where $\mathrm{T}$ is a transition metal and $\mathrm{X}=\mathrm{S}$, Se or Te, the host material is arranged in
XTX sandwiches which are weakly coupled by van der Waals forces. ${ }^{7}$ The intercalant is introduced in the van der Waals gap between the XTX sandwiches. Only electron donor species have been intercalated into transition metal dichalcogenides. Since the lowest lying empty energy levels occur in the $3 d$-bands, intercalation can be used as a controlled means to vary the band filling and the Fermi level. Thus intercalation can produce a semiconductor-metal or metalsemiconductor transition depending on the host, the intercalant species, and intercalant concentration. Changes in band filling can result in Fermi-surface driven instabilities such as charge density waves and the Peierls instability. Transition metals from Groups IV, V, VI, VII, and VIII of the periodic table form layered dichalcogenides. Intercalation can be accomplished with alkali metal atoms ( $\mathrm{Li}, \mathrm{Na}, \mathrm{K}, \mathrm{Rb}, \mathrm{Cs}$ ), noble metals $(\mathrm{Cu}, \mathrm{Ag})$, divalent metals $(\mathrm{Ca}$, $\mathrm{Sr}, \mathrm{Ba})$, column III metals $(\mathrm{Al}, \mathrm{Ga}, \mathrm{In}, \mathrm{Tl})$, transition metals $(\mathrm{V}, \mathrm{Cr}, \mathrm{Mn}, \mathrm{Fe}, \mathrm{Co}, \mathrm{Ni})$, column IV species ( $\mathrm{Si}, \mathrm{Ge}, \mathrm{Sn}, \mathrm{Pb})$, and various organic molecules (such as amines, pyridine, ammonia, hydrazine). ${ }^{7}$

While the fundamental structural unit for the graphite host is an atomic monolayer, and that for the transition metal dichalcogenides is a three-layered sandwich, the alumino-silicate clays (or sheet silicate clays) require as many as seven planes to define a single constituent sheet, making clays relatively rigid against transverse layer distortions. ${ }^{8}$ Clays are intercalated by positively charged cations with or without waters of hydration to form clay intercalation compounds (CICs). Almost every element of the periodic table can be intercalated into clays. Thus, a much larger number of CICs than GICs can be synthesized.

Layered III-VI compounds such as InSe also provide interesting host materials for intercalation. For example, lithium or silver can be intercalated into InSe by a spontaneous reaction in n-butyllithium via an electrochemical process. ${ }^{9}$ This system is of interest as a $\mathrm{Li}$ solid-state battery. The $\mathrm{Li}$ ions in Inse are intercalated on tetrahedral sites, and optical measurements have shown a high degree of in-plane ordering in these intercalation compounds.

\section{Properties}

In the intercalated layered materials, superlattice formation perpendicular to the layer planes arises from the staging phenomenon, which is defined by a periodic arrangement of $n$ layers of host material between sequential intercalate layers, where $n$ is the stage index (see Figure 2). Of the various host materials, graphite exhibits the most pronounced occurrence of staging and the highest degree of staging fidelity. The physical basis for the staging phenomenon in intercalation compounds is the strong interatomic intercalantintercalant binding relative to the binding between the intercalant and the host material, favoring a close-packed in-plane intercalant arrangement. The introduction of each intercalate layer into the host material adds a substantial strain energy as the crystal expands to accommodate the intercalate layer. The resulting interlayer intercalate repulsion favors the insertion of a minimum number of intercalate layers, consistent with a given average intercalate concentration. Thus for a given intercalate concentration, the minimal energy state corresponds to a close-packed in-plane intercalate arrangement with the largest pos-

Continued

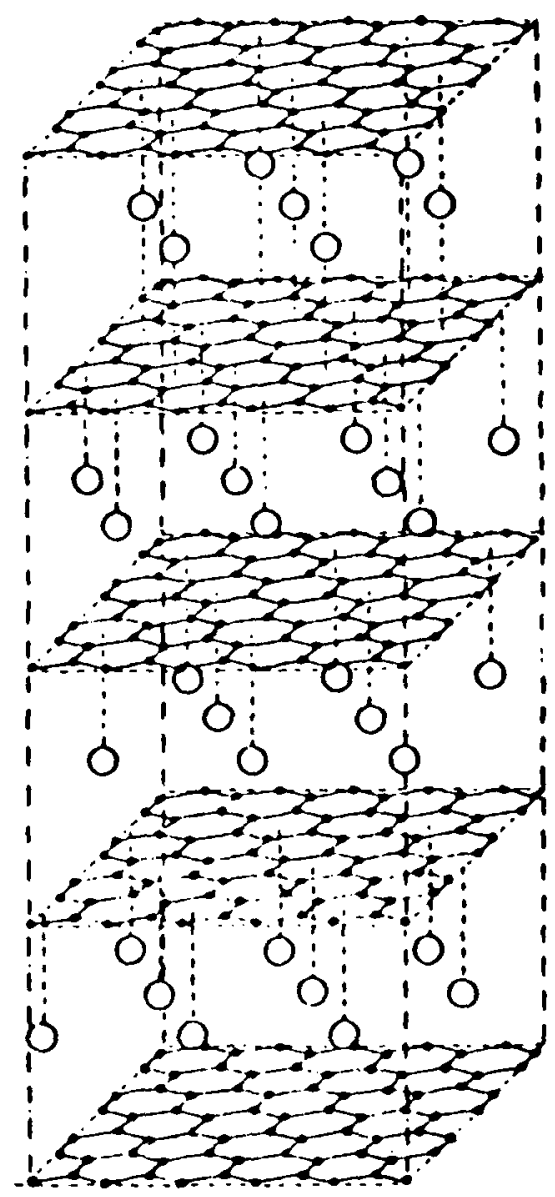

Figure 1: Model for an intercalation compound. Illustrated is the graphite host material (denoted by honeycomb layered sheets) between which monolayers of alkali metal guest species (open circles) are inserted. 


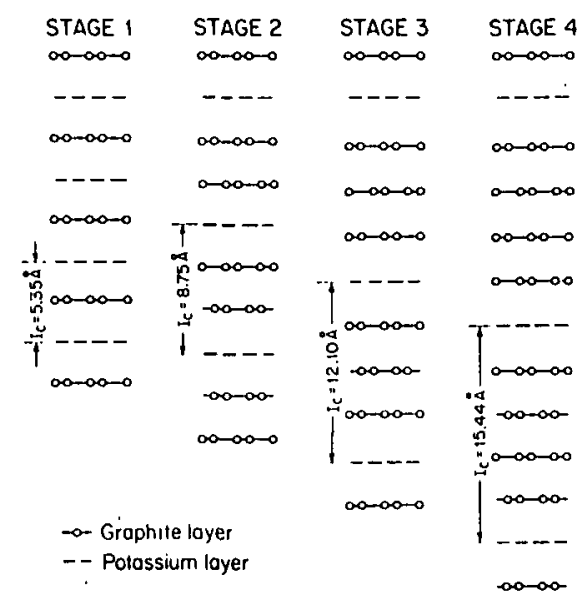

Figure 2: Schematic diagram illustrating the staging phenomenon in K-GICs for stages $1 \geq n \geq 4$. The potassium layers are indicated by dashed lines and the graphite layers by solid lines connecting open circles, and indicating schematically a projection of the carbon atom positions. For each stage, the distance $I_{c}$ between adjacent intercalate layers is indicated.

sible separation between a minimum number of intercalant layers. This combination of conditions results in the staging phenomenon and c-axis superlattice formation. In practice it is possible to prepare single-staged graphite intercalation compounds (GICs) with only small (1-5\%) admixtures of secondary-staged regions. A number of other host materials also exhibit the staging phenomenon, but usually to a lesser degree, consistent with their lower bonding anisotropy. ${ }^{1}$

The simplest method for stage characterization of intercalation compounds is x-ray diffraction based on (00l) reflections. Such measurements show that well-staged GIC materials can be prepared up to stage $n \sim 10$, indicating a repeat distance $I_{c}$ between consecutive intercalate layers of $I_{c} \sim 40 \AA$.

Analysis of $(00 l)$ x-ray diffractograms by means of Bragg's law

$$
l \lambda=2 I_{c} \sin \theta_{l}
$$

shows that the c-axis repeat distance $I_{c}$ for GICs obeys the very simple relation

$$
I_{c}=d_{s}+(n-1) c_{0}
$$

where $d_{s}$ is the distance between two graphitic layers between which the intercalant layer is sandwiched, $n$ is the stage index and $c_{0}(3.35 \AA)$ is the interlayer separation in pristine graphite. Since $d_{s}$ and $c_{0}$ are usually found to be independent of stage, the graphite intercalation compounds can be considered as layered 1D superlattice structures formed by two constituents: the intercalant monolayer or sandwich and the $n$ host material layers where $n$ is the stage index (see Figure 2).
The $(00 l)$ diffractograms yield information on the overall $c$-axis lattice constant $I_{c}=d$, the thickness of the individual constituents (e.g., $d_{1}$ and $d_{2}$ of constituents $M_{1}$ and $M_{2}$ where $d=d_{1}+d_{2}$ ), the in-plane intercalant density as well as the deviations from the superlattice periodicity through analysis of relative line intensities and linewidths, and modeling of the structure factor. For multiple (e.g., three) layer intercalant sandwiches, the $(00 l)$ peak intensities also provide information on the sequencing of the layers and on the layer separation within the intercalate sandwich and the separation between the intercalant and adjacent graphitic layers. For example, the air stable $\mathrm{SbCl}_{5}$-GICs contain a three layer intercalate sandwich structure consisting of an $\mathrm{Sb}$ layer surrounded by two $\mathrm{Cl}$ layers which in turn interface with the graphite bounding layers.

Through controlled layer-by-layer deposition of the constituent species under high vacuum conditions and computer control, ${ }^{10}$ deliberately structured heterostructure superlattices can be prepared (see Figure 3) where $d$ is the superlattice periodicity, comprising a distance $d_{1}$, of material $M_{1}$, and $d_{2}$ of material $M_{2}$. Deliberately structured superlattices can be formed from a large variety of materials $M_{1}$ and $M_{2}$, including: two semiconductors with band offsets of the same sign (Type I-GaAs/ $\mathrm{Al}_{x} \mathrm{Ga}_{1-x} \mathrm{As}$ ) or of different sign (Type IIInAs/GaSb); the same semiconductor (e.g., GaAs) with alternating $n$ - and $p$-type regions ("nipi" superlattice); metal/semiconductor or magnetic metal/semiconductor or superconductor/semiconductor sequences; two different metals or superconductor/ normal metal or magnetic/nonmagnetic sequences of materials; or two different amorphous materials.

In the typical semiconductor superlattice sample (thickness $\sim 1 \mu \mathrm{m}$ ), the periodic unit of distance $d=d_{1}+d_{2}$ is repeated many times (e.g., 100 times). Each period typically varies between a few layers and many layers $(10 \AA$ to $500 \AA)$. Superlattices of these types are today the subject of an extremely active international research field, from both the point of view of fundamental physics and for practical applications.

The characteristics of the layered intercalation compounds and the deliberately structured superlattices are more complementary than similar. Because of the layerby-layer deposition sequence of $\mathrm{MBE}$ or MOCVD, it is possible to form periodic multilayer structures from a very large number of constituents and to make the thickness of the constituent layers as large as might be of interest for a specific application (e.g., $500 \AA$ ). On the other hand, the layer-by-layer insertion characteristic of the intercalation process promotes the introduction of single layers of guest constituents with atomically sharp interfaces, two limits that are very difficult (or per- haps not possible) to achieve with many deliberately structured materials. Even with the constraints of the intercalation process, considerable variation is possible in the intercalated layer thickness, ranging from $0.36 \AA$ for Li in graphite ${ }^{\prime \prime}$ to $-57 \AA$ for n-octadecylamine $\left(C_{18}\right)$ in $\operatorname{TaS}_{2} .{ }^{12}$ Some host materials such as silicate clays acconmodate multiple sequential intercalate layers, though in such cases, staging fidelity is not high. Furthermore, the bi-intercalation process in graphite makes it possible to increase the c-axis separation of a given chemical species; for example, a stage $1 \mathrm{bi}$ intercalation compound consisting of the sequence $\mathrm{G} / \mathrm{CoCl}_{2} / \mathrm{G} / \mathrm{GaCl}_{3} / \mathrm{G}$ allows increased separation between sequential magnetic cobalt layers through the introduction of nonmagnetic $\mathrm{GaCl}_{3}$ layers between alternating graphite $(G)$ layers. ${ }^{13}$

\section{D Superlattices}

Various types of ordering also occur in the two-dimensional layer planes. This ordering can be divided into three main classes: commensurate, incommensurate, and discommensurate ordering. Commensurate ordering with perfect interlayer registry is found at the interface of materials $M_{1}$ and $M_{2}$ in a heterogeneous semiconductor superlattice structure when the lattice constants are matched $a_{1}=a_{2}$ ). The great popularity of $\mathrm{GaAs} / \mathrm{Al}_{x} \mathrm{Ga}_{1-x} \mathrm{As}$ in deliberately structured superlattices is partly due to the high degree of lattice matching of these two constituents and the resulting dislocation-free commensurate interfaces that can be prepared with abrupt compositional discontinuities on an atomic scale. ${ }^{10}$

The strained-layer superlattice supports commensurate ordering at the interface even for materials $M_{1}$ and $M_{2}$ with a lattice constant mismatch of $1 \%$ or more. ${ }^{14}$ In this case the commensurate interface is

Continued

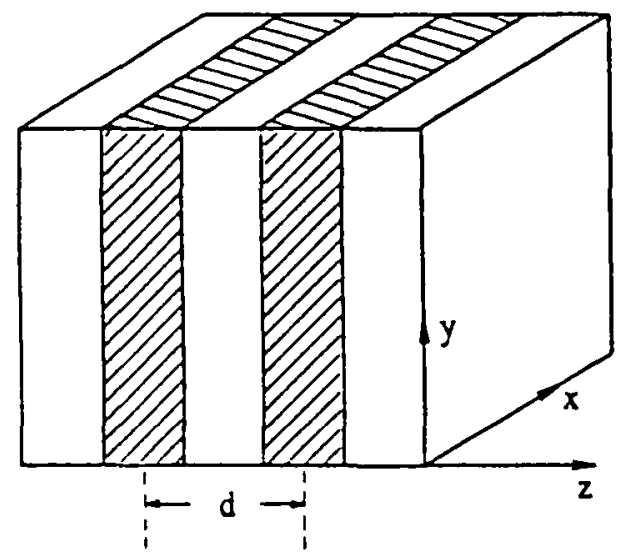

Figure 3: Heterojunction superlattice of periodicity $d$. Each superlattice unit cell consists of a thickness $d_{1}$ of material $M_{1}$ and $d_{2}$ of material $M_{2}$. 


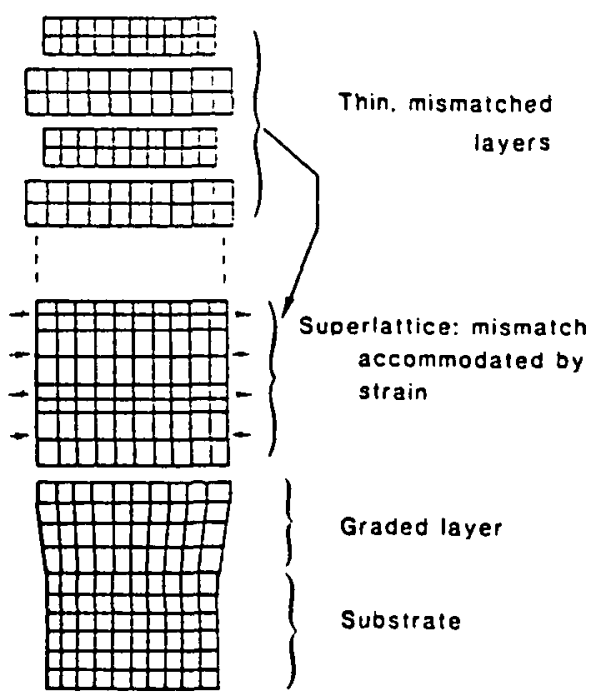

Figure 4: Schematic representation of a strained-layer superlattice. ${ }^{14}$

achieved by stretching thin layers of the smaller lattice constant constituent while compressing thin layers of the larger lattice constant material (see Figure 4). The formation of a commensurate interface in an intercalation compound is closely related to the corresponding structure in a strained-layer superlattice. In the intercalation compound the guest species seek a location at a potential minimum which for the graphite host material is over (or beneath) a hollow in the graphite honeycomb structure.

A second constraint on the intercalant in the intercalation compound is the attractive nearest neighbor force and hard core repulsion that collectively favor an in-plane nearest neighbor separation similar to that in the bulk compound. If both constraints can be approximately satisfied simultaneously with a small stretching or contraction of the intercalant layer, a commensurate intercalation compound results, as shown in Figure 5 for stage 1 potassium intercalated into graphite. ${ }^{5}$

In the context of the strained-layer superlattice, most of the expansion (or contraction) for GICs occurs in the intercalate layer because of the unusually high stiffness of the graphite layers. In contrast, for strained-layer superlattices prepared by $\mathrm{MBE}$, both strained-layer constituents participate significantly in the expansion/ contraction needed to form the commensurate interface.

Another difference between the commensurate interfaces in the MBE strainedlayer superlattices and graphite intercalation compounds relates to the relative sizes of the in-plane unit cells of the two constituents. These unit cells are usually the same for the strained-layer superlattices, but for the graphite intercalation compounds the large size of the intercalant atoms relative to carbon atoms results in an in-plane 2D superlattice in which the inter- calant unit cell area is a multiple (e.g., 3, 4, 7,9 , etc.) of that of the graphite host material, similar to the situation of adsorbed rare gas species on a graphite surface. For example, in the $(2 \times 2)$ superlattice structure of a stage $1 \mathrm{~K}-\mathrm{GIC}$ (Figure 5), the unit cell area of the intercalant is four times greater than that of the graphite host.

In the layer planes, zone folding effects have been observed for commensurate intercalation compounds where the intercalate lattice constant is a multiple of that of the host material. The in-plane zone folding maps $k \neq 0$ points into the zone center, giving rise to new interband transitions for the electronic properties and new phonon modes in the Raman spectra. ${ }^{1}$

From a materials point of view, the formation of commensurate $2 \mathrm{D}$ interfaces permits synthesis of new metastable structures. For example, the $(2 \times 2)$ structure shown in Figure 5 for an alkali metal layer sandwiched between graphite layers does not occur in any bulk alkali metal phase. An example of a metastable structure in a deliberately structured metallic multilayer, is the $\mathrm{Nb} / \mathrm{Zr}$ superlattice where it is possible to grow a phase of bcc $\mathrm{Zr}$ on a bcc $\mathrm{Nb}$ substrate, ${ }^{15}$ in contrast to the hexagonal structure found in bulk crystalline $\mathrm{Zr}$. The study of novel metastable metallic phases is one exciting research area that will be greatly enriched by the wider availability of metallic superlattices and multilayers.

Relatively large lattice mismatches can be accommodated in strained layer superlattices for small thicknesses $d_{1}$ and $d_{2}$ of the constituents. ${ }^{14}$ When the mismatch or layer thicknesses become too large to support lattice accommodation, incommensurate structures with a high density of dislocations are formed. For such interfaces in deliberately structured materials at room temperature, a significant amount of interdiffusion occurs, so that the interfaces become smeared out over several atomic layers normal to the interface. Such incommensurate structures commonly occur in metal superlattices where the two metals have the same crystal structure but a large lattice mismatch, or where the two metals have different crystal structures. ${ }^{16,17}$

For GICs, incommensurate structures occur when there is a large lattice mismatch between the intercalate lattice constants and the possible commensurate structures that are compatible with the graphite honeycomb structure (e.g., $(\sqrt{3} \times \sqrt{3}) \mathrm{R} 30^{\circ}(2 \times 2) \mathrm{RO}^{\circ},(\sqrt{7} \times \sqrt{7}) \mathrm{R} 19.1^{\circ}$, etc). For incommensurate GICs, the lattice constant of the intercalant usually remains essentially unchanged relative to the pristine parent material. In contrast to the smeared out interface for deliberately structured heterostructures, the interface between the graphite and incommensurate intercalant layers is atomically abrupt. Even though the intercalant and the graphite layers have different lattice con- stants, the incommensurate intercalant layers can remain orientationally locked to the graphite, as evidenced by the spot diffraction patterns characteristic of most incommensurate GICs. This phenomenon in incommensurate GICs is yet another manifestation of the independence of positional and orientational ordering in 2D systems. Thus incommensurate intercalation compounds form an interesting, limiting case of deliberately structured superlattices where atomically sharp interfaces can be achieved.

For intermediate cases where some accommodation occurs between the two constituents at an interface, a discommensurate structure is achieved because of significant interactions between atoms in materials $M_{1}$ and $M_{2}$ at the interface. This accommodation is achieved over a commensurate domain, and the lattice mismatch is accomplished by the insertion of an extra lattice plane of the small lattice constant material in the domain walls. As the commensurate domains become smaller, or the domain walls become larger, greater lattice mismatches can be accommodated. When the insertion of the extra lattice planes is periodic, a striped domain phase is achieved. ${ }^{18}$ Discommensuration phenomena have been frequently reported in alkali metal GICs. ${ }^{19}$ Because of the quasi-2D properties of the intercalant layers, much attention has been given to the study of phase transitions in intercalation compounds. ${ }^{19}$

\section{Charge Transfer}

Intercalation causes large changes in electron concentration because of the

Continued

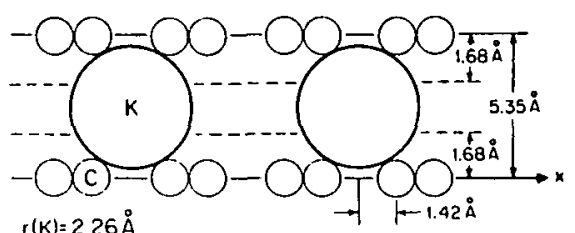

$r(C)=0.71 \AA$

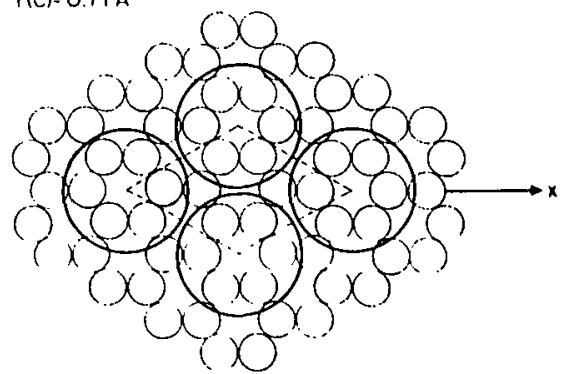

Figure 5: Hard sphere model for the first stage $\mathrm{C}_{8} \mathrm{~K}$ intercalation compound, using the metallic $K$ radius. The upper figure shows an $x-z$ cross section while the lower figure shows an $x-y$ projection. The unit cell for the $(2 \times 2)$ in-plane superlattice is indicated. ${ }^{5}$ 
transfer of charge between the intercalant and host material. Charge transfer introduces electrostatic forces which play a role in the intercalation process and also contribute in a major way to the binding of the intercalation compound. For semiconducting host materials, such as selected transition metal dichalcogenides, intercalation can cause an insulator-metal transition. ${ }^{7}$ For conducting host materials, intercalation usually results in an increase in conductivity, as for graphite host materials.

By transferring carriers from regions of low mobility (the intercalant) to regions of high mobility (graphite), large increases in the electrical conductivity can be achieved, in analogy to the charge transfer process in modulation-doped semiconductor superlattices discussed below. The charge transfer process in donor GICs can be readily understood on the basis of Figure 6. Suppose for example that the intercalant species is the alkali metal potassium. Each potassium atom has one valence electron. If this electron is totally transferred to the graphite layers, the intercalate layer becomes positively charged, attracting electrons to the graphite layers. Thus most of the transferred charge resides in the graphite bonding layers adjacent to the intercalate. Because the in-plane intercalate concentration in a stage 1 compound $\mathrm{C}_{8} \mathrm{~K}$ is $\frac{1}{8}$ of the carbon concentration in a graphite layer, intercalation can achieve as much as a three order of magnitude increase in the carrier concentration.

Modulation doping in deliberately structured materials is completely analogous to charge transfer in intercalation compounds. For a semiconductor heterostructure superlattice such as $\mathrm{GaAs} / \mathrm{Al}_{x} \mathrm{Ga}_{1-x} \mathrm{As}$, the donor impurities are introduced through a shutter in the MBE system while growing the wide gap semiconductor layers, but no donor impurities are introduced into the narrow gap semiconductor layers during their growth. Electron charge is transferred from the large bandgap semiconductor to the small gap semiconductor to achieve a lower electron energy state. Thus the donor defects are only present in the $\mathrm{Al}_{x} \mathrm{Ga}_{1-x} \mathrm{As}$ region, while the carriers are in the GaAs region, which is spatially separated from the donor defects. By this mechanism, very high mobilities can be achieved, since the carriers are confined to the high mobility layers while the ionized impurity scatters are in the wide bandgap layers.

Further tailoring the superlattice structure by inserting undoped $\mathrm{Al}_{x} \mathrm{Ga}_{1-x}$ As layers between the doped $\mathrm{Al}_{x} \mathrm{Ga}_{1-x}$ As layers and the GaAs layers yields even higher mobility materials. The greatest increase in mobility is found at low temperatures, where scattering from ionized impurities is the dominant mechanism for reducing the mobility in uniformly doped material. Improvements in compositional tailoring of

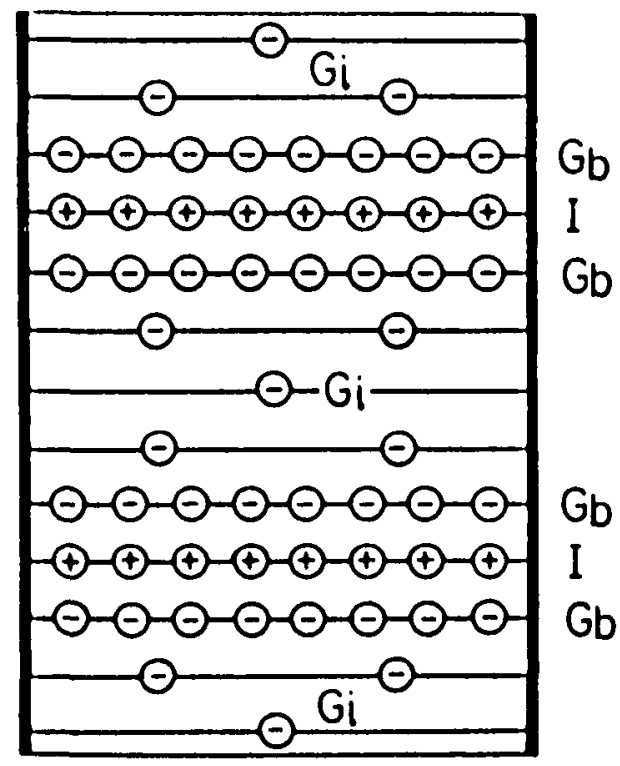

Figure 6: Schematic diagram of electronic charge distribution in the graphite layers of a stage 5 donor compound. I, $G_{b}$, and $G$, refer, respectively, to intercalate, graphite bounding, and graphite interior layers.

the superlattice unit cells have increased by approximately three orders of magnitude the low temperature in-plane mobility within the GaAs region. Low temperature mobility values up to $2 \times 10^{6} \mathrm{~cm}^{2} / \mathrm{Vs}$ have been measured, close to the theoretical limit. ${ }^{20}$ The high in-plane mobility of this GaAs material has been exploited in such devices as modulation-doped field-effect high electron mobility transistors (HEMTs) which have high switching speeds and low power consumption. ${ }^{21}$ Modulated doping can also achieve high mobility p-type material. This is an important technological advance for making possible ultralow power logic based on n- and p-channel transistors, with high transconductance.

Intercalation results in major changes in the transport properties of the host material. ${ }^{1}$ Of the various layers within the unit cell (see Figure 6), the conductivity of the graphite bounding layers (adjacent to the intercalant) is dominant because of the high carrier density in these layers relative to the graphite interior layers, and because of the much higher carrier mobility in the graphite bounding layers relative to the intercalate layer. The in-plane conductance of a GIC can be approximated as a superposition of parallel conductances associated with each type of layer in the unit cell. ${ }^{5}$ Typically, the in-plane electrical conductivity $\sigma_{a}$ of GICs increases from that for graphite by an order of magnitude or more. The magnitude of the increase of the $\sigma_{a}$ depends on the intercalate species and its concentration. Room temperature values of $\sigma_{a}$ exceeding that of copper have been reported for $\mathrm{AsF}_{5}$ intercalated graphite. 22,23
It is believed ${ }^{5,6}$ that the alkali metal intercalants donate essentially all their free carriers to the graphite layers for the higher stage compounds $(n \geq 2)$, though the donated electrons remain predominantly in the graphite bounding layers which then screen the positively charged intercalate layers. A rapid decrease in carrier density occurs with distance into the graphite interior layers. The screening is calculated explicitly through the charge distribution given by self-consistent band calculations.

The charge transfer in donor compounds is often one or two electrons (for monovalent or divalent species) per intercalate unit but in some cases the charge transfer is incomplete. Generally the transfer for donor GICs is greater than that for acceptor compounds. For acceptor compounds, the intercalate layer becomes negatively charged by extracting electrons predominantly from the graphite bounding layers, in contrast to the situation shown in Figure 6 for the donor compounds. Thus a high hole concentration is found in the graphite bounding layers, and the hole concentration decreases rapidly with distance into the graphite interior layers. The electronic charge distribution in the graphite and intercalate layers has been calculated selfconsistently for a number of GICs.

Hall effect measurements in GICs show that the sign of the dominant carrier can be positive (for acceptors) or negative (for donors). Thus intercalation causes the Fermi level to rise (donors) or to fall (acceptors). For many GICs one carrier type predominates, and qualitative results for the carrier density and carrier mobility can be obtained approximately from Hall effect and magnetoresistance measurements through the two carrier type model. ${ }^{5}$

As the temperature $T$ is lowered, the inplane electrical conductivity $\sigma_{a}$ for GICs increases. Quantum oscillatory phenomena can be observed readily in both donor and acceptor GICs at low temperatures. Quantum oscillatory phenomena are also used to study the constant energy surfaces of semiconducting superlattices in deliberately structured materials. ${ }^{10}$

\section{Quasi 20 Magnetism and \\ Superconductivity}

Intercalation compounds and metallic superlattices offer many opportunities and challenges for the study of new physics and new materials science. Some of the new physics involves novel behavior in low dimensional (anisotropic) magnetism and superconductivity. ${ }^{\prime}$ In general, the intercalation of magnetic species into a host material results in a magnetically ordered intercalation compound below a magnetic ordering temperature $T_{r}$. The intercalation of superconducting intercalants generally results in superconducting intercalation compounds. Varying the separation be-

Continued 
tween sequential magnetic layers through the staging mechanism can reduce the interplanar magnetic coupling in a controlled manner to very small values, providing a convenient system for studying the transition between $3 \mathrm{D}$ and $2 \mathrm{D}$ magnetism. Similarly, increasing the separation between intercalate layers can achieve quasi 2D superconductivity in an intercalation compound.

The ability to form atomically sharp interfaces in intercalation compounds allows the explicit separation of individual magnetic layers so that the interlayer exchange coupling can be reduced by several orders of magnitude. Thus intercalation compounds offer special advantages for studying low dimensional magnetism. This property has been exploited in transition metal dichalcogenides, where a large number of different transition metal species can be introduced as monolayers either directly as a constituent of the host material or as an intercalant. ${ }^{7}$ To produce a magnetic superlattice, the GICs are preferred because of their ability to stage.

In addition, by proper choice of intercalant, the spin dimensionality can be varied. As the stage index increases, the interplanar exchange and anisotropy couplings $I^{\prime}$ and $J^{\prime}$ 'become very small, so that the magnetic interactions are largely confined to a single plane and 2D magnetic behavior results. When the intraplanar anisotropy term $J_{A}$ is small, then the exchange couplings are essentially isotropic and a Heisenberg spin system results. If the anisotropy term $I_{A}$ is of comparable magnitude and of opposite sign to the exchange interaction /, then only the $x$ and $y$ spin components are important and the system is described by an $X Y$ model whereby the spins lie in the magnetic planes. Finally, if $I_{A}$ is large and of the same sign as $J$, then the $z$ components of the spin are dominant and the system is described by an lsing model whereby the spins are directed perpendicular to the magnetic planes. As the temperature and magnetic field are varied, unusual magnetic phase transitions are observed for the various types of spin alignments that can arise in these low dimensional systems.

Superlattices formed from deliberately structured magnetic systems offer a wealth of complementary opportunities for magnetic studies. For example, with MBE it is possible to prepare superlattices of magnetic/nonmagnetic conductors (or insulators) or superlattices with constituents exhibiting different types of magnetism (such as AF/F heterostructure superlattices where $A F$ and $F$ denote, respectively, antiferromagnetic and ferromagnetic constituents). One interesting superlattice system that has been studied extensively is the $D y / Y$ system where the Dy forms a spiral spin configuration and the $Y$ is nonmagnetic. Long range coupling of the Dy spins across the nonmagnetic $Y$ layers has been established by study of the magnetic ordering as the layering distances $d_{1}$ and $d_{2}$ of the Dy and $Y$ layers were varied in relation to the magnetic spiral distance. ${ }^{24}$

Because the coherence distances of the superconducting wave function are large compared to interatomic distances, large caxis repeat distances are highly desirable for the study of low dimensional superconductivity. This condition was first achieved by the intercalation of $n$-octadecylamine into $\mathrm{TaS}_{2}$, achieving an intercalated transition metal dichalcogenide with an interlayer repeat distance of $\sim 57 \AA .{ }^{12}$ More recently, detailed and elegant studies of $2 \mathrm{D}$ superconductivity were carried out using a deliberately structured superlattice of $\mathrm{Nb} /$ Ge where the thicknesses of the semiconducting $\mathrm{Ge}$ and superconducting $\mathrm{Nb}$ constituents were varied. ${ }^{16}$ By using $\mathrm{MBE}$ techniques it would be possible to decrease the thickness of the interface so that more quantitative studies of $2 \mathrm{D}$ superconductivity could be carried out. Though no superconducting GICs with c-axis repeat distances $I_{\text {. }}$ greater than the superconducting coherence distance $\xi_{r}$ have yet been synthesized, the atomically sharp interfaces in superconducting GICs offer promise for studies complementary to those made possible by deliberately structured superconducting superlattices. Additional stimulus to these studies will be provided by the recently discovered hightemperature superconductivity $T_{c} \sim 98 \mathrm{~K}$ ) in layered oxide compounds. ${ }^{25}$ At present, the role of the layering in achieving these

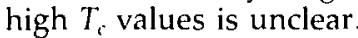

\section{Acknowledgments}

The author thanks Dr. G. Dresselhaus for many important discussions. This work was supported by AFOSR contract \#F49620-83-C-0011.

\section{References}

1. Intercalation in Layered Materials, edited by M.S. Dresselhaus (Plenum Press, New York, 1987), Vol. 148, NATO Series B.

2. Intercalated Graphite, edited by M.S. Dresselhaus, G. Dresselhaus, J. Fischer, and M. Moran, (Mater. Res. Soc. Proc. 20, North-Holland, New York, 1983).

3. Graphite Intercalation Compounds, edited by P. Eklund, M.S. Dresselhaus, and G. Dresselhaus (Mater. Res. Soc. Extended Abstracts, EA-2, Pittsburgh, PA 1984).

4. Graphite Intercalation Compounds, edited by M.S. Dresselhaus, G. Dresselhaus, and S.A. Solin (Mater. Res. Soc. Extended Abstracts, EA-8, Pittsburgh, PA 1986).

5. M.S. Dresselhaus and G. Dresselhaus, Adv. Phys. 30 (1981) p. 139.

6. S.A. Solin. Adv. Chem. Phys. 49 (1982) p. 455

7. W.Y. Liang, in Intercalation in Layered Materials, edited by M.S. Dresselhaus (Plenum, New York, 1987) p. 31.
8. S.A. Solin, in Intercalation in Layered Materials, edited by M.S. Dresselhaus (Plenum, New York, 1987) p. 145.

9. C. Julien, E. Hatzikraniotis, K. Paraskevopoulos, A. Chevy, and M. Balkanski, Solid State lonics, 18-19 (1986) p. 859. 10. Synthetic Modulated Structures, edited by L. Chang and B. Giessen (Academic Press, Orlando, FL, 1985).

11. S. Basu, C. Zeller, P. Flanders, C. Fuerst, W. Johnson, and J. Fischer, J. Mater. Sci. and Eng. 38 (1979) p. 275.

12. F. Gamble and T. Geballe, in Trentise on Solid State Chemistry, edited by N. Hannay (Plenum, New York, 1976) p. 89.

13. A. Hérold, G. Furdin, D. Guérard, L. Hachim, M. Lelaurain, N. Nadi, and R. Vangelisti, Synthetic Metals, 12 (1985) p. 11. 14. G. Osbourn, R. Biefeld, and P. Gourley, Appl. Phys. Lett. 41 (1982) p. 172.

15. W. Lowe and T. Geballe. Phys. Rev. B29 (1984) p. 4961.

16. S. Ruggiero, T. Barbee, Jr., and $M$ Beasley. Phys Rev. B26 (1982) p. 4894.

17. C. Falco and I. Schuller, Synthetic Modulated Structures, edited by L. Chang and B. Giessen (Academic Press, Orlando, FL 1985) p. 339.

18. A. Erbil, A. Kortan, R. Birgeneau, and M.S. Dresselhaus, Phys. Rev. B28 (1983) p. 6329.

19. R. Moret, Intercalation in Layered Materials, edited by M.S. Dresselhaus (Plenum, New York, 1987) p. 185.

20. H. Störmer, Surf. Sci. 132 (1983) p. 519 21. F. Capasso, W. Tsang, and G Williams, IEEE Trans, on Electron Devices, ED-30 (1983) p. 381.

22. F. Vogel, G. Foley, C. Zeller, E. Falardeau, and J. Gan, J. Mater. Sci. and Eng. 31 (1977) p. 261.

23. 1. Shioya, H. Matsubara, and S. Murakami, Synthetic Metals, 14 (1986) p. 113. 24. M. Salamon, S. Sinha, J. Rhyne, J. Cunningham, R. Erwin, J. Borchers, and C. Flynn, Phys. Rev. Lett. 56 (1986) p. 259. 25. K. Kitazawa, Final Program and $A b-$ stracts of the 1986 MRS Annual Meeting. Mater. Res. Soc., Pittsburgh, PA (1986) p. 682, and MRS Bulletin, Vol. XII No. 1 (1987) p. 70; M.K. Wu, J.R. Ashburn, C.J. Torng, P.H. Hor, R.L. Meng, L. Gao, Z.J. Huang, Y.Q. Wang, and C.W. Chu, Phys. Rev. Lett. 58 (1987) p. 908; and MRS BULLETIN, Vol. XII No. 2 (1987) p. 7.

Mildred S. Dresselhaus is Institute Professor and Abby Rockefeller Mauze Professor of Electrical Engineering and Physics at the Massachusetts Institute of Technology. Her research has focused on electronic, optical and magnetooptical properties of semiconductors and semimetals. Her recent work is concentrated on graphite intercalation compounds, graphite fibers, and the modification of electronic materials by intercalation and implantation. She was recently elected to the governing Council of the National Academy of Sciences. 